\title{
ANÁLISE DO PERFIL CLÍNICO E QUALIDADE DE VIDA EM UM GRUPO DE MULHERES ACOMETIDAS DE CÂNCER DE MAMA EM INSTITUIÇÃO DE APOIO DE URUGUAIANA/RS
}

\author{
CLINICAL PROFILE AND QUALITY OF LIFE ANALYSIS OF A GROUP OF WOMEN WITH BREAST CAN- \\ CER IN A SUPPORTING INSTITUTION IN URUGUAIANA, STATE OF RIO GRANDE DO SUL, BRAZIL
}

\section{Teresinha Joseilda da Silva ${ }^{a}$, Luana Paula Navab, Cibeli Ferreira Martins ${ }^{c}$, Fernanda Vargas Ferreira ${ }^{d}$ \\ aterejosz@yahoo.com.br, bluanapn10@yahoo.com.br, ${ }^{c}$ cibeli_martins@yahoo.com.br, danandafvf@gmail.com Universidade Federal do Pampa - Uruguaiana (RS), Brasil}

\section{RESUMO}

Introduçáo: $\mathrm{O}$ câncer de mama e suas terapêuticas têm gerado um impacto sobre a qualidade de vida $(\mathrm{QV})$, uma vez que podem desencadear manifestaçóes clínicas como linfedema, restrição de movimento de ombro e alteraçôes de sensibilidade. Objetivo: Conhecer o perfil clínico e o impacto das condutas terapêuticas sobre a qualidade de vida de mulheres com câncer de mama alocadas em instituição de apoio a pacientes oncológicos de Uruguaiana/RS. Materiais e métodos: Tratou-se de um estudo observacional com abordagem quantitativa entre novembro e dezembro de 2014. As voluntárias foram avaliadas através de anamnese e questionários de QV European Organization for Research and Treatment of Cancer Quality of Life Questionnaire Core30 (EORTC QLQ-C30) e Supplementary Questionnaire Breast Cancer ModuleQLQ (BR-23). Resultados: A maioria das voluntárias era de etnia branca, no pós-menopausa, submetidas a tumorectomias, quadrantectomias, mastectomias e à radioterapia e quimioterapia. As principais queixas foram preocupação com o futuro, alteração na sensibilidade mamária, déficit cognitivo, constipação, sentimento de menor atração física e diminuição da libido. A autopercepção da saúde em geral foi positiva, entretanto, a QV foi avaliada de forma mais impactante pelas tumorectomizadas e quadrantectomizadas. Conclusáo: $\mathrm{O}$ impacto sobre a $\mathrm{QV}$ pode estar relacionado à interferência do estigma de que mastectomias sejam seguras para o controle da doença e prevenção de recidivas, pelo déficit cognitivo e pela ausência de adaptação para o enfrentamento.

Palavras-chave: Capacidade funcional; qualidade de vida; câncer de mama; instrumentos avaliativos.

\section{ABSTRACT}

Introduction: Breast cancer and its treatment have impacted on quality of life (QoL) due to clinical manifestations such as lymphedema, restriction of shoulder movement and sensory changes. Objective: To appraise the clinical profile and the impact of treatment on quality of life of women with breast cancer in a supporting institution in Uruguaiana/RS. Materials and methods: A cross-sectional study was carried out from a quantitative approach in the period November-December 2014. The volunteers were submitted to anamnesis and application of QoL questionnaires such as the European Organization for Research and Treatment of Cancer Quality of Life Questionnaire Core30 (EORTC QLQ-C30) and Supplementary questionnaire Breast Cancer Module - QLQ (BR-23). Results: Most of the volunteers were Caucasian women, post-menopause, undergoing tumorectomy, quadrantectomy and mastectomy, radiotherapy and chemotherapy. Main complaints concerned the future, sensory changes, cognitive impairment, constipation, less physical attraction and lack of sexual desire. Self-perception of health was positive, however, QoL was considered negative by women undergoing tumorectomy and quadrantectomy. Conclusions: Impact on QoL may be related to stigma of mastectomies as safe for illness control and recurrence prevention, by cognitive impairment and lack of adaptation for coping with breast cancer.

Keywords: Functional capacity; quality of life; breast cancer; evaluation tools. 


\section{Introdução}

O câncer de mama ou carcinoma mamário (CM) é o resultado de múltiplas divisões celulares que se reproduzem em grande velocidade e que podem afetar os tecidos vizinhos e provocar metástases. Esse câncer aparece sob a forma de nódulos e, na maioria das vezes, podem ser identificados através do autoexame ${ }^{1}$. Constitui-se como a neoplasia de maior ocorrência entre as mulheres em países desenvolvidos e em desenvolvimento ${ }^{1}$, sendo que o Brasil se encontra entre os países com a mais elevada taxa de incidência.

Quanto ao diagnóstico, os programas de rastreamento e detecção precoce, em sua maioria, se apoiam no autoexame das mamas (AEM), exame clínico das mamas (ECM) e mamografia $(M M G)^{2}$. O ECM se constitui base para a solicitação de exames complementares por meio de inspeção dinâmica/estática e palpação nas regióes torácica e axilar, a partir dos 40 anos. O AEM permite detectar mudanças nas propriedades físicas da mama, tumores e acometimento de linfonodos axilares entre o $7^{\circ}$ e $10^{\circ}$ dia do ciclo menstrual ${ }^{3}$. Os exames de imagem, além da MMG, ultrassonografia (USG) e ressonância magnética $(\mathrm{RM})$ desempenham papel central na detecção e na conduta das doenças mamárias. A MMG identifica lesóes subclínicas ${ }^{4}$. A USG apresenta papel adjuvante à mamografia ou como primeira escolha em situaçóes como gravidez e mulheres jovens ${ }^{5}$. A biópsia do linfonodo sentinela (BLS) apresenta-se como um método de amostragem axilar seletiva, minimamente invasivo e altamente sensível para identificação de metástases. O linfonodo sentinela (LS) é definido como o primeiro gânglio que recebe a drenagem linfática do tumor, na possível rota do $\mathrm{CM}^{6}$. Caso haja a presença de células tumorais, será feita a linfadenectomia axilar (LA), caracterizada pela remoção de um ou mais gânglios linfáticos, para fins de tratamento, indicativo de terapia adjuvante pós-operatória e prognóstico ${ }^{7}$.

Sua etiologia é multifatorial, sendo que em torno de $90 \%$ são esporádicos e apenas $10 \%$ se relacionam a mutaçóes hereditárias nos genes BRCA1 e BRCA2 ${ }^{3}$. Entre os fatores ambientais destacam-se menarca precoce, menopausa tardia, nuliparidade ou primiparidade em idade avançada, maior densidade de tecido mamário, exposição à radiação, ausência ou períodos curtos de amamentaçáo, idade, obesidade, tabagismo e uso de contraceptivos orais ${ }^{8}$.

A cirurgia conservadora que se fundamenta na exérese cirúrgica do tumor (tumorectomia, setorectomia, quadrantectomia); manejo axilar (LS com ou sem EA) e radioterapia (RT) integram a terapêutica locorregional para o controle local do câncer em estádios iniciais9. Dentre as mastectomias, a de Madden consiste na remoção da glândula mamária, aponeurose anterior e posterior do músculo grande peitoral, esvaziamento axilar, com preservação dos músculos grande e pequeno peitoral ${ }^{10}$.

Entre as possíveis condutas terapêuticas se destacam terapias conservadoras como a radioterapia, a quimioterapia e a hormonioterapia e cirúrgicas como tumorectomia, quadrantectomia e mastectomia, que podem ser associadas ou não, dependendo das condiçôes clínicas da paciente ${ }^{11}$.

Como possíveis repercussôes constam limitação da amplitude de movimento do ombro, considerada a mais frequente e a que gera maiores transtornos nas atividades de vida diária. Também são citadas alteraçóes de sensibilidade, linfedema, hiperpigmentação e dispneia ${ }^{12,13}$. Suas repercussôes dependem da extensão da abordagem cirúrgica axilar, realização da radioterapia pós-operatória e de infecçôes ${ }^{14}$.

Baseado na consideração de que as terapias podem gerar impacto sobre a qualidade de vida do indivíduo que é definida como "a percepção do indivíduo de sua posição na vida, no contexto da cultura e sistemas de valores nos quais ele vive e em relação aos seus objetivos, expectativas, padrōes e preocupaçōes"15, a Fisioterapia em Oncologia tem se destacado sob o prisma da promoção de saúde e prevenção em uma equipe multiprofissional, já na fase pré-operatória ${ }^{16,17}$.

Dessa forma, este estudo objetivou conhecer o perfil de um grupo de mulheres com câncer de mama, alocadas em instituição de apoio a pacientes oncológicos de Uruguaiana/RS, e o impacto das condutas terapêuticas sobre a qualidade de vida.

\section{Métodos}

Esta pesquisa se caracterizou como transversal e em abordagem quantitativa, aprovada pelo Comitê de Ética em Pesquisa da Universidade Federal do Pampa (UNIPAMPA) sob o Protocolo no 853.185, de acordo com a Resolução 466/2012, realizada entre novembro e dezembro de 2014 em uma casa de apoio a pacientes oncológicos em Uruguaiana/RS.

O universo populacional foi constituído por mulheres com diagnóstico de câncer de mama, participantes da instituição, e para compor a amostra foram utilizados os critérios de inclusão e exclusão (Figura 1) e aceite do Termo de Consentimento Livre e Esclarecido (TCLE).

Para a efetivação do estudo, realizou-se a divulgação da pesquisa na instituição convidando as voluntárias para participarem do estudo explanando-se objetivos e condutas; após o aceite do TCLE, e da avaliação dos critérios de inclusão e de exclusão, aplicou-se a anamnese para identificação de dados pessoais, ginecológicos, estilos e hábitos de vida a fim de delinear o perfil 
das mulheres. O status foi avaliado por meio da escala Eastern Cooperative Oncology Group (ECOG), que investiga o estado funcional do paciente oncológico, considerando a qualidade de vida e as atividades de vida diária cujos escores são entre 0 e 4, em que 0 representa paciente com atividade normal. A triagem cognitiva foi através do Exame do estado mental (MEEM), tendo como pontos de corte: para analfabetos $\leq 15$ pontos, 1 a 11 anos de escolaridade $\leq 22$ e com escolaridade superior a 11 anos $\leq 27^{18}$.

Figura 1: Organograma dos critérios de inclusão e exclusão das participantes.

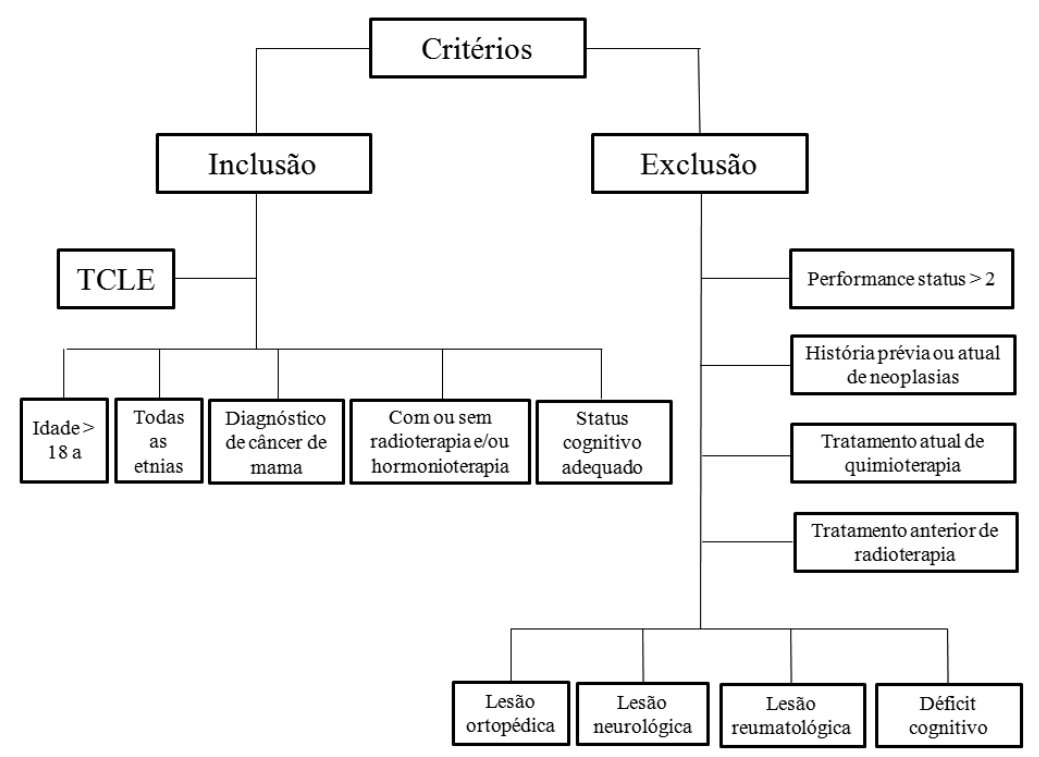

Após, aplicou-se o questionário European Organization for Research and Treatment of Cancer Quality of Life Questionnaire Core30 (EORTC QLQ-C30) composto por 30 itens que incorpora cinco escalas funcionais (desempenho físico, funcional, cognitivo, emocional e social), três escalas de sintomas (fadiga, dor, náusea e vômito) e escalas de qualidade de vida e estado de saúde global. Os itens simples avaliam sintomas adicionais comumente relatados por pacientes com câncer como dispneia, perda do apetite, distúrbio do sono, constipaçáo e diarreia, bem como o impacto financeiro da doença e seu tratamento ${ }^{15}$.

Também se utilizou o Supplementary Questionnaire Breast Cancer Module - QLQ (BR-23) que avalia os efeitos resultantes dos tratamentos, sendo constituído por 23 questóes que se subdividem em duas escalas: escala funcional composta pelas subescalas imagem corporal (BRBI), funcionamento sexual (BRSEF), prazer sexual (BRSEE) e perspectivas futuras (BRFU); e escala sintomática composta pelas subescalas efeitos da radioterapia (BRST), sintomas no braço (BRAS) e preocupações como perda de cabelo (BRHL) ${ }^{17}$.

Para a análise dos dados foram avaliadas as frequências absoluta (n) e relativa (\%) de cada variável e/ou a média \pm desvio-padrão.

\section{Resultados}

Foram entrevistadas 19 mulheres que realizaram intervenção cirúrgica para o câncer de mama, submetidas ou não a tratamentos conservadores, sendo três participantes excluídas por nâo responderem a todos os questionários, totalizando, assim, 16 voluntárias.

Neste estudo, o perfil das voluntárias era de mulheres brancas (9 participantes, $56,25 \%)$, casadas $(7,43,75 \%)$, com nível de educação fundamental incompleto (6, $37,50 \%)$, sedentárias $(16,100 \%)$ e com histórico familiar de neoplasias de mama e de ovários (5, 31,25\%).

Quanto ao perfil uroginecológico, a média de idade da menarca foi de 12,06 anos e a maioria das voluntárias encontravam-se no pós-menopausa (11, $68,75 \%$ ) com média de idade de 51,25 anos. Já, em relação aos tipos de cirurgia, foram realizadas quadrantectomias (em maior número: 7 pacientes, $43,75 \%)$, tumorectomias $(4,25 \%)$ e mastectomias radicais modificadas $(5,31,25 \%)$; as terapias conservadoras realizadas foram radioterapia (14 pacientes, $87,5 \%)$ e quimioterapia (12,75\%), ilustradas na Tabela 1, com o hemicorpo homolateral a cirurgia. Destaca-se também que o tempo de pós-operatório destas mulheres variou de 3 a 10 meses. 
Tabela 1: Características das voluntárias quanto ao perfil uroginecológico, tipo de intervenção cirúrgica, hemicorpo e terapias conservadoras.

\begin{tabular}{|c|c|c|c|}
\hline $\begin{array}{l}\text { Perfil clínico das } \\
\text { participantes }\end{array}$ & $\begin{array}{l}\text { Número/ } \\
\text { frequência }\end{array}$ & média & DP \\
\hline \multicolumn{4}{|l|}{ Idade } \\
\hline 36-38 anos & \multicolumn{3}{|c|}{$16(100 \%) 51,25 \pm 9,94$} \\
\hline \multicolumn{4}{|l|}{ Dados uroginecológicos } \\
\hline $\begin{array}{l}\text { Menarca } \\
8-14 \text { anos }\end{array}$ & \multicolumn{3}{|c|}{$16(100 \%) 12,06 \pm 1,48$} \\
\hline \multicolumn{4}{|l|}{ Período reprodutivo } \\
\hline Ativo & \multicolumn{3}{|c|}{$5(31,25 \%)-$} \\
\hline Inativo & \multicolumn{3}{|c|}{$11(68,75 \%) 47 \pm 4,02$} \\
\hline \multicolumn{4}{|l|}{ Tipos de cirurgia } \\
\hline Mastectomia radical & \multicolumn{3}{|c|}{$5(31,25 \%)$} \\
\hline Quadrantectomia & \multicolumn{3}{|c|}{$7(43,75 \%)$} \\
\hline Tumorectomia & \multicolumn{3}{|c|}{$4(25 \%)$} \\
\hline \multicolumn{4}{|l|}{ Terapias conservadoras } \\
\hline Radioterapia & \multicolumn{3}{|c|}{$14(87,5 \%)$} \\
\hline Quimioterapia & \multicolumn{3}{|c|}{$12(75 \%)$} \\
\hline \multicolumn{4}{|l|}{ Hemicorpo acometido } \\
\hline Direito & \multicolumn{3}{|c|}{$8(50 \%)$} \\
\hline Esquerdo & \multicolumn{3}{|c|}{$8(50 \%)$} \\
\hline
\end{tabular}

Quanto à qualidade de vida no grupo das voluntárias com cirurgias conservadoras (tumorectomia e quadrantectomia) as principais queixas envolveram constipaçáo, cansaço, preocupação, déficit de memória e sensibilidade alterada na mama homolateral. Já no grupo das mastectomizadas, os descontentamentos citados foram: preocupação, diminuição do desejo sexual, alteração na sensibilidade da mama afetada e problemas de pele na regiáo mamária ipsilateral, respectivamente, demonstrado nas tabelas 2 e 3.

Tabela 2: Principais queixas das voluntárias submetidas à tumorectomia e à quadrantectomia.

\begin{tabular}{lcc}
\multicolumn{1}{c}{ Queixas } & Número & Frequência \\
Constipação & 7 & $63,6 \%$ \\
Preocupaçáo & 8 & $72,7 \%$ \\
Déficit de memória & 8 & $72,7 \%$ \\
Sentimento de menor atraçáo física & 7 & $63,6 \%$ \\
Sensibilidade alterada na mama & 8 & $72,7 \%$ \\
\hline
\end{tabular}

Tabela 3: Principais queixas das voluntárias submetidas à mastectomia radical modificada.

\begin{tabular}{lcc}
\multicolumn{1}{c}{ Queixas } & Número & Frequência \\
\hline Diminuição no desejo sexual & 4 & $80 \%$ \\
Preocupação & 4 & $80 \%$ \\
Sensibilidade alterada na mama & 3 & $60 \%$ \\
$\begin{array}{l}\text { Problemas de pele na região mamária } \\
\text { afetada* }\end{array}$ & 4 & $80 \%$ \\
\hline
\end{tabular}

Legenda: *ardência, coceira e pele seca.
Ao se considerar a autopercepção sobre a saúde em geral, no grupo das cirurgias mais conservadoras, a saúde em geral variou de 5 a 7 e a qualidade de vida de 4 a 7; ao passo que, no grupo das mastectomizadas, a primeira variou de 6 a 7 e a segunda de 6 a 7 , conforme a Tabela 4.

Tabela 4: Percepção da saúde em geral e qualidade de vida das voluntárias.

\begin{tabular}{|c|c|c|c|}
\hline $\begin{array}{c}\text { Tumorectomia / } \\
\text { Quadrantectomia }\end{array}$ & Pontuaçáo & Número & Frequência \\
\hline \multicolumn{4}{|l|}{ Saúde em geral* } \\
\hline & 7 & 6 & $54,5 \%$ \\
\hline & 6 & 3 & $27,2 \%$ \\
\hline & 5 & 2 & $18,1 \%$ \\
\hline \multicolumn{4}{|l|}{ Qualidade de vida** } \\
\hline & 7 & 5 & $45,4 \%$ \\
\hline & 6 & 3 & $27,2 \%$ \\
\hline & 5 & 2 & $18,1 \%$ \\
\hline & 4 & 1 & $9,09 \%$ \\
\hline \multicolumn{4}{|l|}{ Mastectomia } \\
\hline \multicolumn{4}{|l|}{ Saúde em geral* } \\
\hline & 7 & 3 & $60 \%$ \\
\hline & 6 & 2 & $40 \%$ \\
\hline \multicolumn{4}{|l|}{ Qualidade de vida** } \\
\hline & 7 & 3 & $60 \%$ \\
\hline & 6 & 2 & $40 \%$ \\
\hline
\end{tabular}

Legenda: ${ }^{*}$ = varia de 1 a 7 (péssima a ótima); ${ }^{* *}=$ varia de 1 a 7 (péssima a ótima).

\section{Discussão}

Quanto às características do grupo de estudo, os achados apresentaram consonância com pesquisas que apontam que o câncer de mama acomete principalmente mulheres na faixa dos 50 anos e da etnia branca ${ }^{11,14,16}$. Uma possível explanação, a idade é o fator de risco mais importante para o CM, 77\% dos casos de CM, diagnosticados nos Estados Unidos entre o período de 1994 e 1998, ocorreram em mulheres com idade igual ou superior a 50 anos de idade ${ }^{2}$. Fato este também observado em um estudo realizado no Brasil, em que o câncer de mama é a principal causa de óbito entre as mulheres na faixa etária de 40 a $69 \operatorname{anos}^{17}$.

Em relação às intervençóes terapêuticas, preconiza-se a preservaçáo da mama para câncer de mama inicial no controle locorregional da doença, o que está de acordo com os resultados deste estudo, uma vez que a maioria das participantes realizou abordagem cirúrgica conservadora e foi submetida à radioterapia ${ }^{16,17}$.

Como resultado pode haver a perda parcial ou total da mama, e as complicações advindas dos tratamentos alteram a biomecânica postural, podendo ser transitórias 
ou permanentes, caracterizadas por anteriorização da cabeça, protrusão de ombro, acentuação das curvas da coluna, elevação do ombro e escápula ipsilateral, rotaçáo da pelve e inclinaçấo da cabeça ${ }^{19}$. Outro fator extremamente importante é a limitaçáo na amplitude de movimento do membro superior, incidindo desfavoravelmente na realização das atividades de vida laborais e de lazer ${ }^{20}$.

Ademais, atualmente, recomenda-se a menor ressecção possível, visto que a mama, para a mulher, não é somente um órgão de adorno ou estímulo sexual e, sim, a representaçáo de sua feminilidade e de sua condição de mulher ${ }^{21}$, bem como para gerar menor déficit físico-funcional ${ }^{22,23 .}$ Outra possível explanação são os programas de rastreamento e detecção precoce do $\mathrm{CM}$, em sua maioria apoia-se no autoexame das mamas (AEM), exame clínico das mamas (ECM) e mamografia (MMG), sendo fator determinante do prognóstico da doença ${ }^{2}$.

Em relação à sintomatologia, ambos os grupos relataram sentimentos de preocupação com o futuro, o que permite teorizar que o contato com o mundo externo gera uma série de fantasias e medos, implicando uma mudança de comportamento social ${ }^{11}$. Ainda, é importante considerar que mesmo quando há o mínimo de ressecçáo cirúrgica, como na tumorectomia e na quadrantectomia $^{24}$, existem relatos de medos e crises, corroborando os dados deste estudo. Também se pode teorizar que a preocupação pode decorrer do processo saúde-adoecimento, já que este envolve distintas condutas terapêuticas e seus respectivos efeitos adversos, chance de cura e possível recorrência, o que tende a afetar as atividades de vida diária (AVDs), laborais e de lazer da mulher com câncer de mama ${ }^{25}$.

Outra complicação em comum envolveu alteração na sensibilidade da mama ipsilateral, achado frequente no pós-operatório e/ou tratamento conservador das neoplasias mamárias ${ }^{26}$, podendo ser na porção medial do braço, axilar e/ou lateral do tórax resultando em anestesia, hipossensibilidade, hipersensibilidade e/ou parestesia ${ }^{27}$. Os principais mecanismos causais seriam relacionados à manipulação e/ou lesão do trajeto do nervo intercostobraquial (NICB) durante a cirurgia ou pela radioterapia sobre o nervo braquial ${ }^{28}$, o que pode justificar o achado tanto em mulheres submetidas a cirurgias mais extensas quanto com menor ressecção; entretanto, ressalta-se que este estudo não avaliou a sensibilidade sensitiva dos trajetos dos nervos NICB e braquial.

Ainda no contexto da região da mama afetada, apenas as mastectomizadas relataram complicaçóes como pele seca e coceira, possivelmente, efeitos advindos da radioterapia como as radiodermites, embora, a maioria das tumorectomizadas e quadrantectomizadas tenha sido submetida também a essa terapia locorregional. Nesse sentido, pode-se justificar o achado pela maior exposiçáo da pele e da prega mamária devido à ablação total da mama ${ }^{29}$ e a outros fatores como esquema de fracionamento, volume de tecido irradiado e radiossensibilidade ${ }^{30}$.

Dentre os sintomas gastrointestinais se destacou no grupo das tumorectomizadas e quadrantectomizadas a queixa de constipação, geralmente relacionada à quimioterapia ${ }^{31}$, o que pode justificar o resultado, uma vez que a maioria das voluntárias foi submetida à terapia sistêmica. Também como possível explanação pode ser citado o hipoestrogenismo associado a mudanças anatômicas e fisiológicas ocorridas no pós-menopausa que comprometem o assoalho pélvico e os esfíncteres ${ }^{32}$, em concordância com o período reprodutivo da maioria das participantes. Ademais, deve-se contextualizar que a constipação também se relaciona à dieta alimentar $\mathrm{e}$ à prática regular de atividade física ${ }^{33} \mathrm{em}$ concordância com os achados deste estudo em que todas as voluntárias eram sedentárias.

Ainda que não sejam conhecidas, pesquisas sugerem que a administração de drogas antineoplásicas (quimioterápicos) pode gerar déficits cognitivos na memória, raciocínio, atenção e aprendizado em $75 \%$ dos sujeitos $^{34}$, o que pode justificar o achado deste presente estudo. Dentre os mecanismos pós-quimioterapia se citam a barreira hematoencefálica, já que os fármacos podem lesar neurônios do hipocampo, região responsável pela função cognitiva normal ${ }^{35}$, danos ao $\mathrm{DNA}^{34}$, disfunçôes na liberação de citocina que, por sua vez, regula o processo inflamatório ${ }^{36}$, diminuição do reparo neuronal e alteraçôes genéticas de neurotransmissores como dopamina que atua nas funçóes executivas e de memória $^{34}$. Outra possível justificativa seria o efeito do envelhecimento sobre o status cognitivo, bem como outros fatores como condição prévia cognitiva e interações hormonais ${ }^{34}$.

Em relação à possível alteração na vida sentimental e sexual após as condutas terapêuticas, as voluntárias sinalizaram impacto negativo sobre o sentimento de atração física e libido, respectivamente, nos grupos de menor e maior ressecção cirúrgica.

A partir desses achados se citam algumas consideraçóes: a) a tensão nervosa e o estresse advêm de possíveis sentimentos de desamparo, de uma perda de vínculo afetivo e sexual e de autoestima ${ }^{37}$ sem uma relação direta com a ablaçáo da mama (total ou parcial), uma vez que, neste estudo, as mastectomizadas não se queixaram de déficit na atração física, todavia, ressalta-se uma diferença numérica entre os grupos, o que pode justificar a ausência dessa complicação; b) o autoconceito de si está diretamente relacionado ao grau de satisfação que a mulher tem com o seu corpo, uma vez que a perda da mama ou parte dela reflete-se no processo de reelaboração da identidade feminina ${ }^{38}$, o que pode explicar a 
dificuldade citada pelas tumorectomizadas e quadrantectomizadas; c) a perda de interesse pode ser um mecanismo de defesa e uma forma de autoproteção diante do embaraço sentido pelo novo corpo $^{39}$; d) a queixa de menor libido pelas mastectomizadas pode advir do choque com a nova realidade em virtude de que muitas mulheres vivenciam a cirurgia e o pós-operatório como um processo de "mutilação" 38 e e) a quimioterapia pode interferir na produção de hormônios sexuais favorecendo disfunçóes como decréscimo na libido ${ }^{39}$.

Estudos enfatizam que a perda da mama é tratada pelas mulheres como um evento traumático, e que associado à retirada parcial ou total da mama ocorre a extirpação da sexualidade, do desejo, do sentimento de feminilidade e da atratividade. Podendo gerar pacientes mais inseguras, ansiosas e deprimidas em relação a sua vida sexual, após o procedimento cirúrgico ${ }^{40}$.

Ademais, é importante destacar que outros possíveis fatores interferentes sobre as queixas sejam os efeitos do climatério sobre a sexualidade, já que a depleção estrogênica afeta a lubrificação vaginal, assim como o contexto de que a sexualidade é complexa e dependente da interação de reaçóes neuroendócrinas/vasculares, de um aparelho psíquico e de um sistema sociocultural ${ }^{14}$.

Outra possível explanaçáo, em relaçáo à queixa de menor desejo sexual, é que as voluntárias encontram-se na faixa etária de 40 a 60 anos, na qual ocorre a passagem da fase reprodutiva para ondas de calor, suores noturnos, insônia, depressão, irritabilidade, ressecamento vaginal, redução no desejo sexual ${ }^{42}$.

No que tange à autopercepção sobre a saúde em geral, ambos os grupos sinalizaram de "boa para ótima", possivelmente, pela influência do padrão de personalidade e do meio cultural no qual estão inseridas ${ }^{43}$. Também se sugere que, após os tratamentos cirúrgicos e/ou conservadores, há uma tendência a se enfatizar os resultados positivos, com sentimentos de esperança e de sentido para a vida, buscando-se afastar os pensamentos negativos ${ }^{44}$.

Considerando-se a qualidade de vida (QV) que é um construto subjetivo e multidimensional ${ }^{16,17,21}$, observou-se que o grupo das tumorectomizadas e quadrantectomizadas considerou-a de "regular a ótima", em contraste à literatura, já que na mastectomia, pela extirpaçáo total da mama, usualmente se observam reflexos mais negativos sobre a autoimagem e a autoestima ${ }^{45}$, além de um possível eliciamento de autorrejeição e frustração ${ }^{46}$, todavia, neste estudo, a percepçáo foi de "muito boa a ótima”. Outra possível explanação é que a QV pode melhorar com a formação de uma equipe multiprofissional que trabalhe de forma integrada e mantenha um relacionamento satisfatório com a paciente ${ }^{47}$. Outra possível justificativa está relacionada às queixas relatadas por ambos os grupos, em relação à alteração de sensibilidade alterada na mama, a intervenção fisioterapêutica, reeducação sensitiva pode ser representada por técnicas manuais realizadas por um profissional ou pelo próprio paciente, sob orientação ${ }^{48}$.

Dessa forma, como possíveis justificativas para o resultado inesperado, teoriza-se que para o grupo de menor extensão cirúrgica possíveis dúvidas quanto à sua identidade sexual e adaptação à doença tenham atuado como fatores interferentes sobre a QV, bem como pela "crença" de que a mastectomia seria uma conduta mais segura para o tratamento do câncer, pois, haveria a "limpeza" do corpo das células malignas, prevenindo-se, assim, recidivas ${ }^{39}$. Ademais, a QV se relaciona à cognição ${ }^{34}$, sendo o déficit cognitivo uma das principais queixas das tumorectomizadas e quadrantectomizadas, o que pode também explanar o resultado.

\section{Conclusão}

Este estudo verificou que a maioria das voluntárias era branca, pós-menopáusicas, submetidas a ressecçóes mais conservadoras e à radioterapia e à quimioterapia.

Como principais queixas se destacaram preocupação com o futuro, alteração na sensibilidade mamária, déficit cognitivo, constipação, sentimento de menor atração física e diminuiçáo da libido.

As voluntárias apresentaram uma autopercepção da saúde em geral positiva, ao passo que a qualidade de vida foi avaliada de forma mais negativa pelas tumorectomizadas e quadrantectomizadas, em contraste à literatura que aponta que quanto maior a extensão cirúrgica, maior o impacto sobre o construto. Esses resultados sugerem a influência do estigma de que a mastectomia é mais efetiva, do déficit cognitivo relatado pelas voluntárias, bem como pela não adaptação à nova condição.

Ressalta-se que esses resultados e discussóes não podem ser generalizados tendo em vista o número de voluntárias, bem como pela complexidade do tema e suas vertentes.

Por fim, sugere-se maior divulgação da abordagem fisioterapêutica sobre a qualidade de vida em mulheres com câncer de mama com vistas a um maior reconhecimento e aprofundamento da temática.

\section{Referências}

1. Bregagnol RK, Dias AS. Alteraçóes funcionais em mulheres submetidas à cirurgia de mama com linfadenectomia axilar total. Rev Bras Câncer. 2010;56(1):25-33.

2. Bastiton AP. Detecção precoce do câncer de mama: conhecimento e prática de mulheres e profissionais da estratégia da saúde da família em Dourados/MS [tese de doutorado] [Internet]. Goiás: Universidade Federal de Goiás e 
Universidade Federal de Mato Grosso do Sul; 2009 [acesso em 2015 nov. 20]. Disponível em: http://bdtd.bce. unb.br/tedesimplificado/tde_arquivos/6/TDE-2009-0911T161250Z-4317/Publico/2009_AdrianePiresBatiston. pdf.

3. Dantas ELR, Sá FHL, Carvalho SMF, Arruda AP, Ribeiro EM, Ribeiro EM. Genética do câncer hereditário. Rev Bras Câncer. 2009; 55(3):263-9.

4. Davim RMB, Torres GV, Cabral MLN, Lima VM, Souza MA. Autoexame de mama: conhecimento de usuárias atendidas no ambulatório de uma maternidade escola. Rev LatAm Enferm. 2003;11(1):21-7.

5. Godinho ER, Koch HA. Rastreamento do câncer de mama: aspectos relacionados ao médico. Radiol Bras. 2004;37(2):91-9.

6. Coelho-Oliveira A, Rocha ACP, Gutfilen B, Pessoa MCP, Fonseca LMB. Identificaçáo do linfonodo sentinela no câncer de mama com injeção subdérmica periareolar em quatro pontos do radiofármaco. Radiol Bras. 2004;37(4):233-7.

7. Swenson KK, Nissen MJ, Ceronsky C, Swenson L, Lee MW, Tuttle TM. Comparison of side effects between sentinel lymph node and axillary lymph node dissection for breast cancer. Ann Surg Oncol. 2002;9(8):745-53.

8. Cesar PGC, Fonseca FLA, Gehrke FS, Alves BCA, Kuniyoshi RK, Giglio AD. Utilização de plataforma gênica no prognóstico do câncer de mama. Arq Bras Ciên Saúde. 2012;37(3):154-61.

9. Gebrim LH, Shida JY, Mattar A, Madeira M. Indicaçóes da cirurgia conservadora no câncer de mama. Femina. 2010;38(11):594-7.

10. Pereira CMA, Vieira EORY, Alcântara PSM. Avaliação de Protocolo de Fisioterapia aplicado a pacientes mactetomizadas a Madden. Rev Bras Cancerol. 2005;51(2):143-8.

11. Duarte T, Andrade A. Enfrentando a mastectomia: análise dos relatos de mulheres mastectomizadas sobre questóes ligadas à sexualidade. Estudos de Psicologia. 2003;8(1):155-63.

12. Marcucci FCI. O papel da fisioterapia nos cuidados paliativos a pacientes com câncer. Rev Bras Cancerol. 2005;51(1):67-77.

13. Gomide LB. Repercussóes tardias da radioterapia sobre a função pulmonar em pacientes com câncer de mama. [dissertação de mestrado] [Internet]. Ribeirão Preto: Universidade de São Paulo; 2006 [acesso em 2015 nov 20]. Disponível em: http://www.teses.usp.br/teses/disponiveis/17/17145/ tde-19122006-145040/pt-br.php

14. Lahoz MA, Nyssen SM, Correia GN, Garcia APU, Driusso P. Capacidade funcional e qualidade de vida em mulheres pós-mastectomizadas. Rev Bras Cancerol. 2010; 56(4): 423-430.

15. Santos ALA, Moura JFP, Santos CAAL, Figueiroa JN, Souza AI. Avaliação da qualidade de vida relacionada à saúde em pacientes com câncer do colo do útero em tratamento radioterápico. Rev Bras Cancer. 2012;58(3):507-15.

16. Gutiérrez MGR, Bravo MM, Chanes DC, Vivo MCR,
Souza GO. Adesão de mulheres mastectomizadas ao início precoce de um programa de reabilitação. Acta Paul Enferm. 2007;20(3):249-54.

17. Jammal MP, Machado ARM, Rodrigues LR. Fisioterapia na reabilitação de mulheres operadas por câncer de mama. $\mathrm{O}$ Mundo da Saúde. 2008;32(4):506-10.

18. O'keeffe ST, Mulkerrin EC, Naveem K, Varughese M, Pillay I. Use of serial Mini-Mental State Examinations to diagnose and monitor delirium in elderly hospital patients. J Am Geriatr Soc. 2005;53(5):867-70.

19. Barbosa JAN, Amorim MHC, Zandonade E, Delaprane ML. Avaliação da postura corporal em mulheres com câncer de mama. Rev Bras Ginecol Obstet. 2013;35(5):215-20.

20. Rezende LF, Beletti PO, Franco RL, Moraes SS, Gurgel MSC. Exercícios livres versus direcionados nas complicaçôes pós-operatórias de câncer de mama. Rev Assoc Med Bras. 2006;1(52):37-42.

21. Monsanto F, Lança C, Sá AC, Coelho CM, Carolino E. Influência do tratamento de radioterapia na qualidade de vida dos doentes com cancro de mama. Saúde \& Tecnologia. 2013;9:40-4.

22. Bergmann A, Mattos IE, Koifman RJ. Fatores de risco para linfedema após câncer de mama: uma revisão da literatura. Fisioter Pesqui. 2008;15(2):207-13.

23. Petito EL, Nazário ACP, Martinelli SE, Facina G, Gutiérrez MGR. Aplicação de programa de exercícios domiciliares na reabilitação do ombro pós-cirurgia por câncer de mama. Rev Lat-Am Enferm. 2012;20(1):35-43.

24. Simeão SFAP, Landro ICR, De Conti MHS, Gatti MAN, Delgallo WD, De Vitta A. Qualidade de vida em grupos de mulheres acometidas de câncer de mama. Cien Saúde Colet. 2013;18(3):779-88.

25. Almeida AM, Mamede MV, Panobianco MS, Prado MAS, Clapis MJ. Construindo o significado da recorrência da doença: a experiência de mulheres com câncer de mama. Rev Latino-am Enferm. 2001;9(5):63-9.

26. Nogueira EA, Bergmann A, Paixão E, Thuler LCS. Alteraçóes sensitivas, tratamento cirúrgico do câncer de mama e nervo intercostobraquial: revisão da literatura. Rev Bras Cancer. 2010; 56(1): 85-91.

27. Bergmann A, Koifman RJ, Koifman S, Ribeiro MJP, Mattos IE. Upper limb lymphedema following breast cancer surgery: prevalence and associated factors. Lymphology. 2007;40:96-106.

28. Santos MSM, Panobianco MS, Mamede MV, Meirelles MCCC, Barros VM. Sensibilidade tátil no membro superior de mulheres submetidas à linfonodectomia axilar por câncer de mama. Rev Bras Ginecol Obstet. 2009;31(7):361-6.

29. Pires AMT, Segreto RA, Segreto HRC. Avaliação das reaçóes agudas da pele e seus fatores de risco em pacientes com câncer de mama submetidas à radioterapia. Rev Latino-Am Enferm. 2008;16(5):1-5.

30. Porock D. Factors influencing the severity of radiation skin and oral mucosal reactions: development of a conceptual framework. Eur Cancer Care. 2002;11(1):33-43. 
31. Nicolussi AC, Sawada ON. Qualidade de vida de pacientes com câncer de mama em terapia adjuvante. Rev Gaúcha Enferm. 2011;32(4):759-66.

32. Oliveira SCM, Pinto-Neto AM, Góes JRN, Conde DM, Santos-Sá D, Costa-Paiva L. Prevalência e fatores associados à constipaçáo intestinal em mulheres na pós-menopausa. Arq Gastroenterol. 2005;42(1):24-9.

33. Collete VL, Araújo CL, Madruga SW. Prevalência e fatores associados à constipação intestinal: um estudo de base populacional em Pelotas, Rio Grande do Sul, Brasil, 2007. Cad Saúde Pública. 2010;26(7):1391-402.

34. Untura LP, Rezende LF. A função cognitiva em pacientes submetidos à quimioterapia: uma revisão integrativa. Rev Bras Cancer. 2012;58(2):257-65.

35. Koppelmans V, Breteler MM, Boogerd W, Seynaeve C, Gundy C, Schagen SB. Neuropsychological performance in survivors of breast cancer more than 20 years after adjuvant chemotherapy. J Clin Oncol. 2012; 30(10):1080-6.

36. Ahles TA, Saykin AJ. Candidate mechanisms for chemotherapy-induced cognitive changes. Nat Rev Cancer. 2007;7(3):192-201.

37. Moura FMJSP, Silva MG, Oliveira SC, Moura LJSP. Os sentimentos das mulheres pós-mastectomizadas. Esc Anna Nery Rev Enferm. 2010;14(3):477-84.

38. Azevedo FR, Lopes MLR. Merleau-ponty e a compreensão da vivência de mulheres mastectomizadas em uso de prótese. Rev Enferm UERJ. 2005;13(2):188-93.

39. Rebelo V, Rolim L, Carqueja E, Ferreira S. Avaliação da qualidade de vida em mulheres com cancro da mama: um estudo exploratório com 60 mulheres portuguesas. Psicologia, Saúde \& Doenças. 2007;8(1):13-32.

40. Cesnik VM, Santos MA. Mastectomia e sexualidade: uma revisão integrativa. Psicologia: Reflexão e Crítica. 2012;25(2):339-49.

41. Rohden F. Diferenças de gênero e medicalizaçáo da sexualidade na criação do diagnóstico das disfunçóes sexuais. Rev Estud Fem. 2009;17(1):89-109.

42. Figueiredo TC, Frigo LF. Climatério e menopausa: um olhar a cerca da sexualidade. Fórum de Fisioterapia. 2012.

43. Al-Ghazal SK, Fallowfield L, Blamey RW. Comparison of psychological aspects and patient satisfaction following breast conserving surgery, simple mastectomy and breast reconstruction. Eur J Cancer. 2000;36(15):1938-43.

44. Pereira SG, Rosenhein DP, Bulhosa MS, Lunardi VL, Lunardi Filho WD. Vivências de cuidados da mulher mastectomizada: uma pesquisa bibliográfica. Rev Bras Enferm. 2006;59(6):791-5.

45. Rossi L, Santos MA. Repercussóes psicológicas do adoecimento e tratamento em mulheres acometidas pelo câncer de mama. Psicologia Ciência e Profissão. 2003;23(4):32-41.

46. Ornelas FA, Rodrigues JRP, Uemura G. Análise sensitiva convencional nos pós-cirúrgico de câncer de mama. Rev Bras Mastol. 2009;19(2):53-9.

47. Meneghim LA, Bortolan S. Menopausa e Terapia de Reposição Hormonal; 2009.

48. Thornton AA. Perceiving benefits in cancer experience. J Clin Psy Med Settings. 2002;9(2):153-65.

\section{Como citar este artigo:}

Silva TJ, Nava LP, Martins CF, Ferreira FV. Análise do perfil clínico e qualidade de vida em um grupo de mulheres acometidas de câncer de mama em instituição de apoio de Uruguaiana/RS. Rev. Aten. Saúde. 2016;14(48):68-75. 